\title{
RÉSEAU SOCIAL NUMÉRIQUE ET IMAGES VECTORIELLES : INTRODUCTION À UNE COMMUNICATION À VOCATION INTERNATIONALE
}

\begin{abstract}
Marilou Kordahi ${ }^{1}$
Dans le contexte de la mondialisation, nous fondons ce travail préliminaire sur l'émergence de la relation entre les Réseaux Sociaux Numériques (RSN) et les systèmes artificiels de communication visuelle, à savoir la signalétique. Cette dernière sert à donner une information sur un sujet, pour faciliter la communication entre les usagers à l'échelle internationale. Ce système de communication visuelle a également une visée pragmatique : il doit conduire le destinataire à accomplir une action et/ ou il doit influer sur sa perception de la réalité. Le signagramme, qui est de type figuratif, est son unité d'écriture. Notre objectif est, tout d'abord, de concevoir un nouveau prototype d'un RSN de communication à vocation internationale, en nous servant de la signalétique et de l'outil de traduction automatique de syntagmes en signagrammes. Le SignaComm est l'intitulé de notre RSN : il est spécialisé et informatif. Ensuite, nous développons ce prototype en vue de tester ses capacités de communiquer des messages visuels à des usagers nationaux et internationaux. En guise d'exemple, nous traitons le cas d'une secousse sismique appartenant au domaine des risques et catastrophes naturels.
\end{abstract}

Le présent projet de recherche a vu le jour en observant la nécessité de communiquer dans un monde globalisé, avec des langues et des

1 Marilou Kordahi, Équipe CITU, Laboratoire Paragraphe, UFR MITSIC, Université Paris 8. Faculté de Gestion et de Management, Université Saint-Joseph de Beyrouth.

Recherches en communication, $\mathrm{n}^{\circ} 42$ (2016). 
cultures diverses et dans des situations pouvant être d'une complexité nouvelle. La recherche d'une communication visuelle utilisant la puissance des Réseaux Sociaux Numériques (RSN) semble être une voie fructueuse que nous proposons d'explorer.

Nous contribuons à la discipline des Sciences de l'Information et de la Communication en explorant l'émergence de la relation entre les systèmes artificiels de communication visuelle et les Réseaux Sociaux Numériques (RSN). À partir des années 1925, un certain nombre de systèmes artificiels de communication visuelle ont vu le jour afin d'améliorer la gestion et l'échange d'informations, en ne tenant pas compte d'un langage naturel spécifique (par exemple, Universal Playground, Istotype) (Takasaki \& Mori, 2007 ; Neurath, 1974). Quant aux dispositifs internationaux de signalisation routière et maritime se servent de systèmes de communication visuelle statique pour transmettre des directives, des risques ou des consignes aux personnes (Vaillant 1999). Dans nos travaux de 2016 et 2013, nous nous sommes intéressées à la signalétique, système artificiel de communication visuelle à vocation internationale, qui sert à donner une information sur un sujet précis (Bertin, 1970). Le signagramme, qui est de type figuratif (Klinkenberg, 1996), est son unité d'écriture (figure 1). Il est une image vectorielle (au format SVG).

D’une manière générale, une image vectorielle est une collection de courbes, comme les courbes de Bézier. L'utilisation des équations mathématiques de ces courbes permet de créer des graphiques vectoriels à l'échelle et permet de les manipuler de façon répétée sans modifier leur qualité.

(Di Mascio et al., 2010, p. 709-710).

Selon Barnes, "le réseau social est en grande partie un système de liens entre des paires de personnes qui considèrent l'autre comme approximativement à égalité sociale »(Barnes, 1954, p. 44). Le terme communauté virtuelle est attribué à l'ouvrage du même titre Les communautés virtuelles par Rheingold \& Lumbroso (1995). Une communauté virtuelle en ligne est un système d'informations artificiel et numérique qui est formé d'un réseau social de membres (Barnes, 1954). Les interactions de cette communauté virtuelle peuvent être nominatives (par exemple, entre deux usagers particuliers) ou bien groupées (par exemple, entre un usager et un groupe spécifique). Les 
usagers de la communauté virtuelle en ligne appartiennent à différents espaces géographiques, cultures, groupes d'âge et groupes sociaux (Wellman, 2001). Le motif d'une communauté virtuelle en ligne est de permettre à un ensemble d'usagers de communiquer à une échelle nationale et internationale (Fernback, 1997 ; Wellman \& Gulia, 1999). L'échange d'informations se fait essentiellement par le biais de la messagerie instantanée, le courriel et l'enregistrement vocal.

Un réseau social numérique est une forme d'application de la communauté virtuelle en ligne (Wellman \& Gulia, 1999; Rheingold \& Lumbroso, 1995). Il est un système artificiel d'informations (par exemple, un site Web dédié à la traduction automatique collaborative) qui utilise les applications basées sur le Web 2.0 (par exemple, les médias sociaux) afin de construire des relations sociales entre des personnes (ou des organisations), partageant des intérêts, des activités, des contacts de la vie réelle (Rainie \& Wellman, 2012 ; Ellison, 2007). La construction de ces relations sociales ne peut avoir lieu et exister qu'une fois ces personnes (ou organisations) se sont inscrites au RSN et par suite en sont devenues membres (Balagué \& Fayon, 2012 ; Cardon, 2009 ; Ellison, 2007).

Notre finalité est, tout d'abord, d'explorer la possibilité de concevoir un premier prototype d'un RSN de communication à vocation internationale, en nous servant de la signalétique (Kordahi, 2013a) et de l'outil de traduction automatique de syntagmes en signagrammes (Kordahi \& Baltz, 2015). Ensuite, nous développons ce prototype afin de tester ses capacités techniques de transmettre des messages visuels à des usagers, indépendamment d'un langage naturel particulier.

"Le SignaComm », qui est l'intitulé de notre RSN, est spécialisé (Ellison, 2007) et informatif (Ma et al., 2011). Il effectue trois tâches consécutives, automatiques (sans l'intervention humaine) et en temps réel. Il traduit le texte d'entrée d'un membre en signagrammes, afin de transmettre le résultat obtenu à un autre membre (figure 4). Ensuite, il affiche l'historique des résultats transmis sous la forme de messages instantanés en signagrammes, dans la page d'échange entre membres (figure 4). Enfin, il montre la position géographique de ses membres (figure 3). Les informations, générées par les utilisateurs et l'établissement en question, doivent être sauvegardées sur les serveurs distants et sécurisés de ce dernier (Di Gangi \& Wasko, 2009). Des utilisateurs de tout âge, de toute culture, de tout milieu social et souffrant de handicap se serviraient du SignaComm. 
À notre connaissance, les projets de recherche traitant le sujet des réseaux sociaux numériques de la signalétique sont limités. Cependant, des travaux de recherche sont menés sur les RSN et la messagerie instantanée combinés à la traduction, notamment ceux de Wong et al. (2012), Yang \& Lin (2010) et Seme (2001). Nous nous servirons d'une part de cet ensemble de travaux publiés et d'autre part, de nos travaux publiés (Kordahi \& Baltz, 2015 ; Kordahi, 2013a, 2013b) afin d'affiner notre direction de recherche et de concevoir le premier prototype du SignaComm.

Le SignaComm pourrait être intégré dans la structure du système d'informations d'une entreprise ou d'un organisme public appartenant à un certain domaine, à savoir le domaine de la logistique (par exemple, le commerce en ligne), l'informatique (par exemple, le service de courrier électronique), de l'éducation (par exemple, le programme de l'enseignement participatif). Nous nous appuierons ici sur le cas d'une situation d'urgence appartenant au domaine des risques et catastrophes naturels. Nous optons d'étudier le cas d'une secousse sismique qui se manifesterait en France du Sud-est, région sismique particulièrement sensible.

Nous aborderons la conception du SignaComm en nous penchant sur les principes de la signalétique (Kordahi, 2013a), de l'architecture modulaire (Parnas, 1972) et du transfert sémantique (Emele et al., 2000). Les règles de la signalétique concernent celles de ses signagrammes. Chaque signagramme est formé d'une " forme externe » (y compris le contour) et d'une "forme interne » (Kordahi, 2013a) (figure 1). La construction des signagrammes repose sur des formes graphiques simples (Neurath, 1974) en évitant les différences linguistiques (Takasahi \& Mori, 2007 ; Takasaki, 2006). Les signagrammes présentés ici sont spécifiquement basés sur nos travaux publiés en 2013 et 2012 (Kordahi, 2013a, 2012). Nous avons choisi de nous appuyer sur les travaux de Desessard et al. (2015) et la banque d'images « Fotolia» pour concevoir et développer environ cent signagrammes. Nous avons utilisé les symboles graphiques, disponibles dans cette banque d'images, qui sont actuellement utilisés à l'échelle internationale. En outre, les couleurs et les formes des signagrammes créées suivre la charte des panneaux routiers (Desessard et al., 2015). Nous avons utilisé cette charte parce qu'elle est toujours en cours d'usage et est assez bien connue à l'échelle internationale.

Selon Parnas (1972), l'architecture modulaire est un principe qui permet de former un système de sous-systèmes informatiques, 
ou modules, indépendants. Un ensemble d'instructions programmées permet de mettre en relation automatiquement ces modules. Le modèle de transfert sémantique, quant à lui, se comporte comme un système de réécriture qui traite un texte d'entrée d'une langue source et construit un nouveau texte de sortie d'une langue cible en utilisant des règles linguistiques et les dictionnaires de langues naturelles (Emele et al., 2000).

Nous expliquerons l'approche suivie pour construire l'architecture conceptuelle et technique du SignaComm pour la prévention des risques en cas de séisme. La démarche suivie pourrait être aussi utilisée pour l'élaboration d'autres représentations de cas. Notre travail est composé de quatre sections. Dans la première, nous expliquerons la position du SignaComm. Dans la deuxième, nous proposerons de concevoir l'architecture du SignaComm que nous appliquerons à ce cas social (celui la prévention des risques en cas de séisme). Puis, nous mènerons un test technique pour évaluer notre RSN. Enfin, nous discuterons de l'ensemble ainsi élaboré avant de conclure notre recherche.

\section{Présentation de la position du SignaComm}

\subsection{Définition du SignaComm}

Le rôle du SignaComm n'est point de représenter la réalité telle qu'elle se présente et encore moins de combler des lacunes dans le monde réel (Proulx \& Latzko-Toth, 2000). Le rôle de ce RSN est de faciliter la création et le partage de messages visuels, récurrents dans la vie réelle (par exemple, des faits actuels) (Wellman \& Gulia, 1999), à une échelle nationale ainsi qu'internationale (Wellman, 2001 ; Fernback, 1997).

La présente définition du SignaComm suit celle de la communauté virtuelle « hybride », décrite par Proulx \& Latzko-Toth (2000). Selon les chercheurs, une communauté virtuelle « hybride » est une « hybridation $d u$ réel et $d u$ virtuel, ou plus exactement l'immanence du virtuel dans le réel, et une conception du réel dans laquelle l'actuel et le virtuel sont en interrelation circulaire et productive » (Proulx \& Latzko-Toth, 2000, p. 104). Étant donné qu'un RSN est une forme d'application de la communauté virtuelle en ligne (Wellman \& Gulia, 1999; Rheingold \& Lumbroso, 1995), alors le SignaComm peut prendre la forme d'une communauté virtuelle en ligne « hybride».

Notre RSN est utilisé pour transmettre des informations structurées quand elles doivent être assimilées rapidement par ses membres (par 
exemple, la communication de consignes préventives pour la prévention des risques). Nous suggérons aussi de nous servir du SignaComm quand les usagers parlent diverses langues ou souffrent de certaines limites (par exemple, l'apprentissage guidé dans le domaine de l'informatique) (Wandmacher et al., 2008).

\subsection{SignaComm, pour une communication à vocation internationale}

Plusieurs applications en ligne, à savoir les RSN et les communautés virtuelles en ligne, utilisent actuellement des systèmes artificiels de communication visuelle afin de faciliter les interactions et les échanges de connaissances entre les différents usagers (Lin \& Ishida, 2014 ; Bell et al., 2015). Ce choix se justifie d'une part, par l'observation des évolutions sociales mondiales (Mattelart, 2000) et d'autre part, par la visée d'un grand public international (Lin \& Ishida, 2014 ; Wellman, 2001). Par conséquent, nous notons que la présence de ces systèmes artificiels de communication se manifeste essentiellement au niveau de l'interface « homme-machine » de ces applications en ligne et de leur dictionnaire d'émoticônes.

L'interface des RSN obéit aux principes de la conception universelle des interfaces numériques, à savoir la simplicité, la flexibilité et l'accessibilité de l'usage (Spiliotopoulos et al., 2012 ; Kobsa et al., 2001). De plus, cette interface est de type graphique et contextuel (Jain et al., 2013). Nous entendons par le terme « interface contextuelle», une interface qui est programmée pour s'adapter aux comportements et aux actions contextuels de l'usager. La nature graphique de l'interface des RSN se fonde sur un modèle de création répondant à des règles bien définies et précises pour assurer un produit homogène et uniforme. Ces règles sont les suivantes : un contenu simple et figuratif, une unicité des représentations graphiques et une unicité de la couleur du contenu (Hartmann, 2008). Quant au dictionnaire d'émoticônes, il contient les symboles des émotions, qui sont en cours d'usage à l'échelle mondiale (Vaillant, 1999).

Jusqu'à présent, nous n'avons pas trouvé de travaux publiés traitant le sujet de la normalisation d'un système artificiel de communication visuelle dédié à l'ensemble des RSN. D'ailleurs, un bon nombre d'entreprises développent leur propre système de communication et l'intègrent dans l'interface des RSN (par exemple, l'interface graphique de WhatsApp) et des outils de la nouvelle technologie (par exemple, l'iPad de Apple) afin de tenter de guider intuitivement et contextuellement les divers usagers dans leurs actions (Jain et al., 2013). Il est pertinent 
de signaler que chaque entreprise (ou organisme) choisit d'adapter la charte de son système de communication visuelle du RSN et de ses outils de la nouvelle technologie correspondants, en fonction de la culture, des usages et des mœurs des pays qu'elle (ou qu'il) cible. Les applications des réseaux sociaux numériques (les interfaces graphiques et dictionnaires d'émoticônes inclus) sont modifiées afin d'une part, être compatibles avec les normes des différents pays, qui souhaiteraient autoriser leurs exploitations par les utilisateurs et, d'autre part, répondre au mieux aux besoins ainsi qu'à l'expérience de ces usagers (Jain et al., 2013 ; Balagué \& Fayon, 2012).

(a)

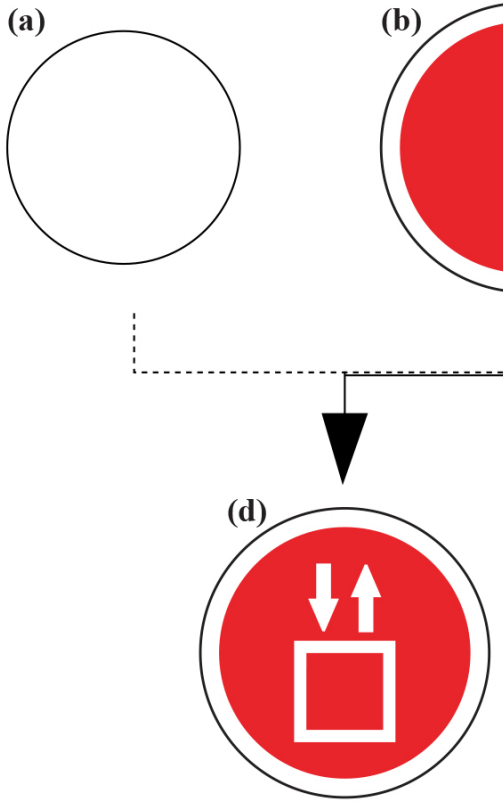

(c)

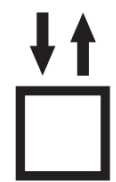

(a) Forme externe P. ex., « verbe » : ne pas utiliser

(b) Contour forme externe

(c) Forme interne

P. ex., « nom commun » : ascenseur

(d) Signagramme

P. ex., syntagme : ne pas utiliser l'ascenseur

Figure 1 : exemple de signagramme

Le SignaComm a les capacités de tendre vers un RSN de communication à vocation internationale à condition de se servir des deux éléments suivants : le système de communication de la signalétique (Kordahi, 2013a) (figure 1) ainsi que l'interface graphique et contextuelle (Jain et al., 2013) répondant aux principes de la conception universelle des interfaces numériques (Spiliotopoulos et al., 2012). Ces principes nous permettront de concevoir au mieux l'interface du SignaComm en vue d'améliorer sa perception et sa compréhension spontanée par tout 
usager. Cependant, le bon avancement du développement du service du SignaComm est en relation avec celui de la signalétique (Kordahi, 2013a), des RSN (Rainie \& Wellman, 2012) et des outils de la nouvelle technologie ainsi qu'avec le pouvoir d'adaptation des usagers. Cette interrelation rend le SignaComm dépendant des trois facteurs que sont les environnements sociaux (Hartmann, 2008), techniques et humains (Proulx \& Latzko-Toth, 2000). Par conséquent, cette dépendance pourrait causer le ralentissement du bon développement du SignaComm et de celui de la réalisation de son objectif. Pour le moment, nous ne traiterons pas l'aspect émotionnel ainsi que les dictionnaires d'émoticônes étant donné que ce RSN est informatif.

Dans la figure 1, nous montrons un exemple d'application de signagramme représentant le syntagme " ne pas utiliser l'ascenseur » (Prévention des risques majeurs ; Desessard et al., 2015).

\section{Architecture graphique du SignaComm : première approche dans la situation-type}

Nous nous inspirons de l'interface graphique du service WhatsApp afin de concevoir l'architecture du SignaComm. Ce travail de conception se fait en deux étapes qui sont les suivantes : l'identification des besoins fonctionnels ainsi que la conception de l'architecture graphique du SignaComm.

\subsection{Identification des besoins fonctionnels du SignaComm}

Quand l'enquête marketing est disponible, cette étude des besoins pourrait être menée en se servant des outils d'aide à la conception de produits ou de services, notamment la matrice de Développement des Fonctions Qualités (QFD). Par exemple, cette matrice servira à définir les attentes des usagers et ensuite, à proposer des solutions adaptées pour y répondre au mieux.

Dans le présent projet, l'identification des fonctionnalités du SignaComm se fait manuellement en analysant les besoins potentiels des usagers (Kobsa et al., 2001). Cette analyse nous permet de distinguer les besoins d'une interface ainsi que de cinq fonctionnalités principales. Cette interface sert à afficher les informations résultantes de l'échange entre les membres du SignaComm, et de l'interaction entre le système du RSN et ses membres. Nous donnons les exemples de la visualisation des messages composés et reçus, des informations personnelles du 
profil d'un membre, et du résultat de la traduction automatique d'un texte écrit en une séquence de signagrammes.

Quant aux fonctionnalités principales, elles prennent la forme de boutons signagrammes dont le rôle est d'initier des événements préalablement programmés. Ces événements sont cinq et sont les suivants : " accéder à la liste des membres » (par exemple, éditer le profil personnel), « traduire le message multilingue » (par exemple, traduire automatiquement un syntagme écrit en un signagramme), " réinitialiser la requête » (par exemple, réinitialiser la traduction automatique d'un syntagme), « envoyer le message » (par exemple, transmettre le résultat de la traduction automatique à un membre) et « afficher la géolocalisation » (par exemple, afficher la position géographique d'un membre). Le bouton-signagramme " traduire le message multilingue » est une nouvelle fonctionnalité que nous créons pour répondre à l'objectif du RSN. Les quatre autres fonctions sont fréquemment utilisées par un grand nombre de RSN internationaux, notamment Facebook, WhatsApp. Nous suggérons de nous servir de ces fonctionnalités existantes pour commencer la création des nôtres.

\subsection{Conception de l'architecture graphique du SignaComm}

La conception de l'architecture du SignaComm comprend celle des pages de navigation, de l'interface graphique et des boutons signagrammes. Nous proposons de constituer le SignaComm de deux pages et d'une fenêtre pop-up (ou fenêtre surgissante), à savoir la page des membres, la page d'échange entre les membres (figure 4) et la fenêtre de géolocalisation d'un membre (figure 3). La navigation entre ces pages se fait à travers les boutons signagrammes. Cette conception est adoptée pour utiliser un nombre de pages limitées en vue d'accéder rapidement à l'information, faciliter l'actualisation instantanée des données des usagers afin d'assurer au mieux l'exactitude des informations, et apporter de la flexibilité de l'usage pour initier une interaction intuitive (Spiliotopoulos et al., 2012).

Étant donné que le SignaComm tend vers un RSN à vocation internationale, nous avons préféré créer une interface uniquement graphique (Spiliotopoulos et al., 2012). Cette interface est composée d'une partie principale et d'une autre secondaire. La partie principale permet de visualiser le contenu des pages du RSN. La partie secondaire, elle, joue le rôle de barre de navigation. Pour le moment, elle contient seulement le bouton signagramme « accéder à la liste des membres ». 
Quant aux boutons signagrammes, nous nous appuyons sur les règles de création des signagrammes (Kordahi, 2013a) et sur les symboles graphiques, publiés dans la banque d'images "Fotolia », pour concevoir leur squelette. Nous nous servons de ces symboles graphiques, car ils sont en cours d'usage sur le marché international. La figure 2 illustre les boutons signagrammes vectoriels qui traduisent les syntagmes suivants : « accéder à la liste des membres », " envoyer le message », " afficher la géolocalisation » et « traduire le message multilingue ».

(a)

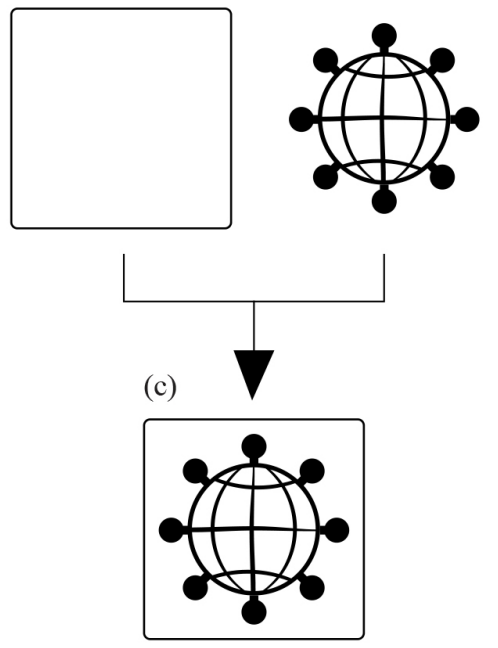

(b)
À gauche :
(a) Forme externe : traduire
(b) Forme interne : message multilingue
(c) Signagramme : traduire le message multilingue A droite, de haut en bas : accéder à la liste des membres; envoyer le message ; afficher la géolocalisation.
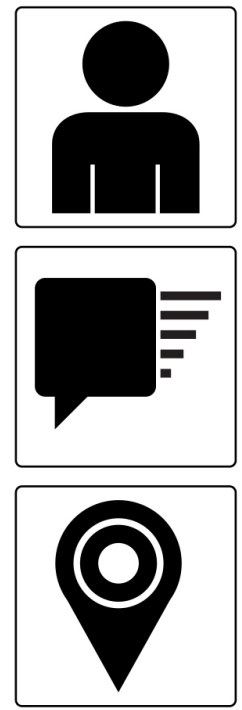

Figure 2 : exemple de boutons signagrammes

\section{Architecture modulaire du SignaComm : premier test technique}

Le SignaComm est écrit avec les langages de programmation PHP, MySQL, Java et XML pour être interrogé à partir d'une page 
Web et être exécuté sur tous les types de plate-forme de la nouvelle technologie. Nous nous servons de la plate-forme Elgg pour développer techniquement notre RSN que nous sauvegardons, à présent, sur un serveur local.

Les trois principaux modules du SignaComm sont le module du profil de l'utilisateur, celui de la traduction automatique d'un texte écrit en une série de signagrammes (Kordahi \& Baltz, 2015) et celui de la messagerie instantanée (Seme, 2001). L'interface est une page Web au format HTML pour permettre l'interaction entre l'usager et le RSN.

\subsection{Module du profil de l'utilisateur}

Ce module principal effectue trois tâches essentielles. Il permet l'inscription d'un utilisateur et l'authentification d'un membre au SignaComm (Ellison, 2007), qui sont une condition primordiale pour se servir de cet outil. L'inscription et l'authentification sont gérées automatiquement et contrôlées par les programmeurs ${ }^{1}$.

Les données sont enregistrées dans la base de données de ce module (la base de données est une collection de données structurées et mises à jour en temps réel). L'inscription est nominative : elle se fait en soumettant un identifiant et un mot de passe ainsi que les données personnelles de l'usager, choisissant de partager ses informations (Yang \& Lin, 2010 ; Cardon, 2009) et celles de sa position géographique, avec le RSN (Khanna, 2015). Les données personnelles sont statiques (tels que les nom et prénom, l'adresse physique, le courriel et le mode d'affichage du profil (privé ou public)) (Proulx, 2012 ; Cardon, 2009) alors que les données de la localisation géographique sont dynamiques. L'authentification d'un membre se fait en soumettant uniquement l'identifiant et le mot de passe préalablement enregistrés.

La deuxième fonction permet à un membre du SignaComm d'y inviter un autre utilisateur en lui envoyant une invitation électronique (par exemple, un message instantané) (Granovetter, 1983) en se servant des deux autres modules.

Ce module est relié à un procédé de géolocalisation (Barrington et al., 2015 ; Khanna, 2015). Celui-ci permet d'effectuer la troisième tâche

1 Les administrateurs et programmeurs ont des droits additionnels. Ils peuvent effectuer des opérations supplémentaires, telles que la validation de l'inscription et la réinscription d'un membre, la modification des échanges entre les membres. $\mathrm{La}$ liste des membres déjà inscrits ainsi que les données personnelles d'un membre sont accessibles à partir de la «page des membres". 
qui est d'une part, de détecter et d'afficher la position géographique de chaque membre du réseau social numérique et d'autre part de lui proposer automatiquement une langue naturelle de conversation. La figure 3 représente la position géographique exacte du membre correspondant, durant cet échange.

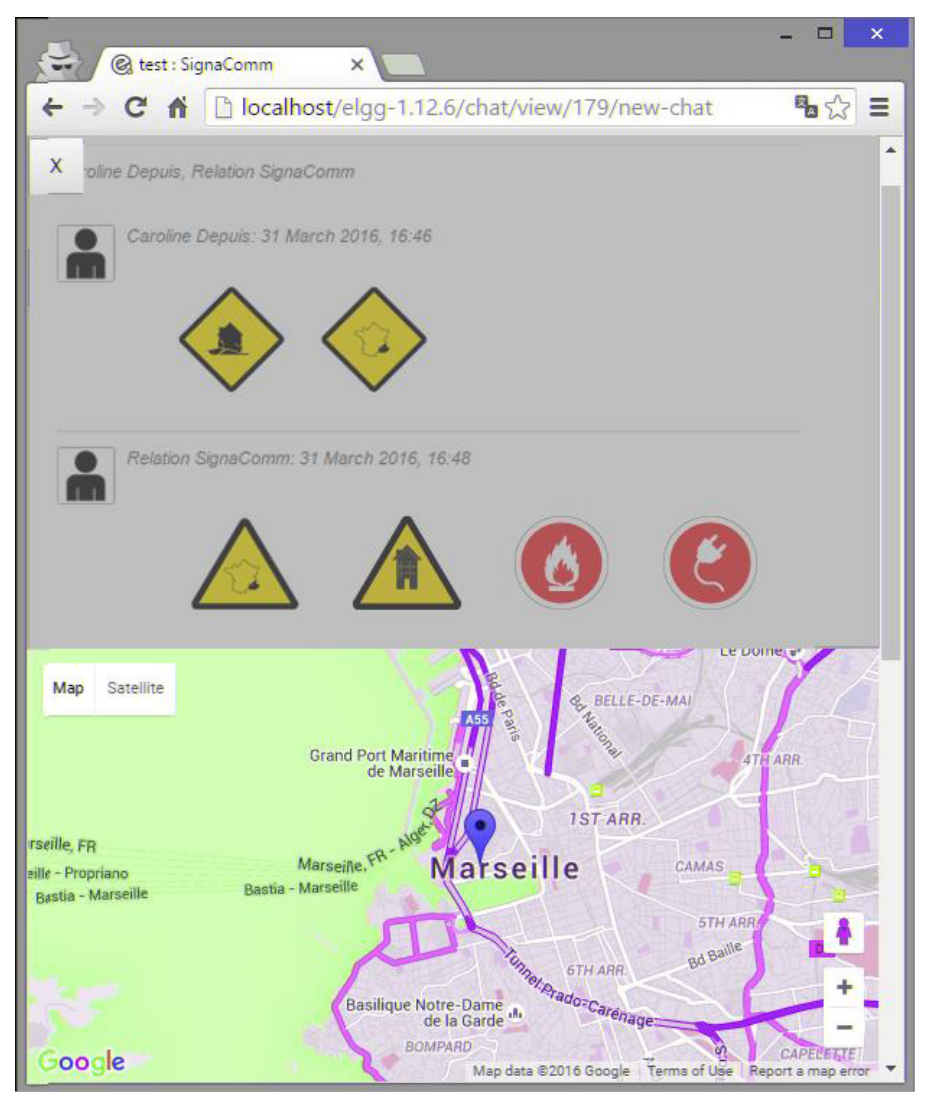

Figure 3 : exemple d'application du procédé de géolocalisation

\subsection{Module de la traduction sémantique}

Nous nous appuyons sur nos travaux de 2015 (Kordahi \& Baltz, 2015) pour intégrer le module de traduction automatique dans la structure du SignaComm. La structure de ce module se fonde sur le dictionnaire de signagrammes pour la prévention des risques liés à une secousse sismique (notre étude de cas) (Kordahi, 2013b), l'ontologie 
liée à la même situation sociale (Provitolo et al., 2009) et une fonction qui traduit le texte d'entrée en signagrammes (Kordahi \& Baltz, 2015).

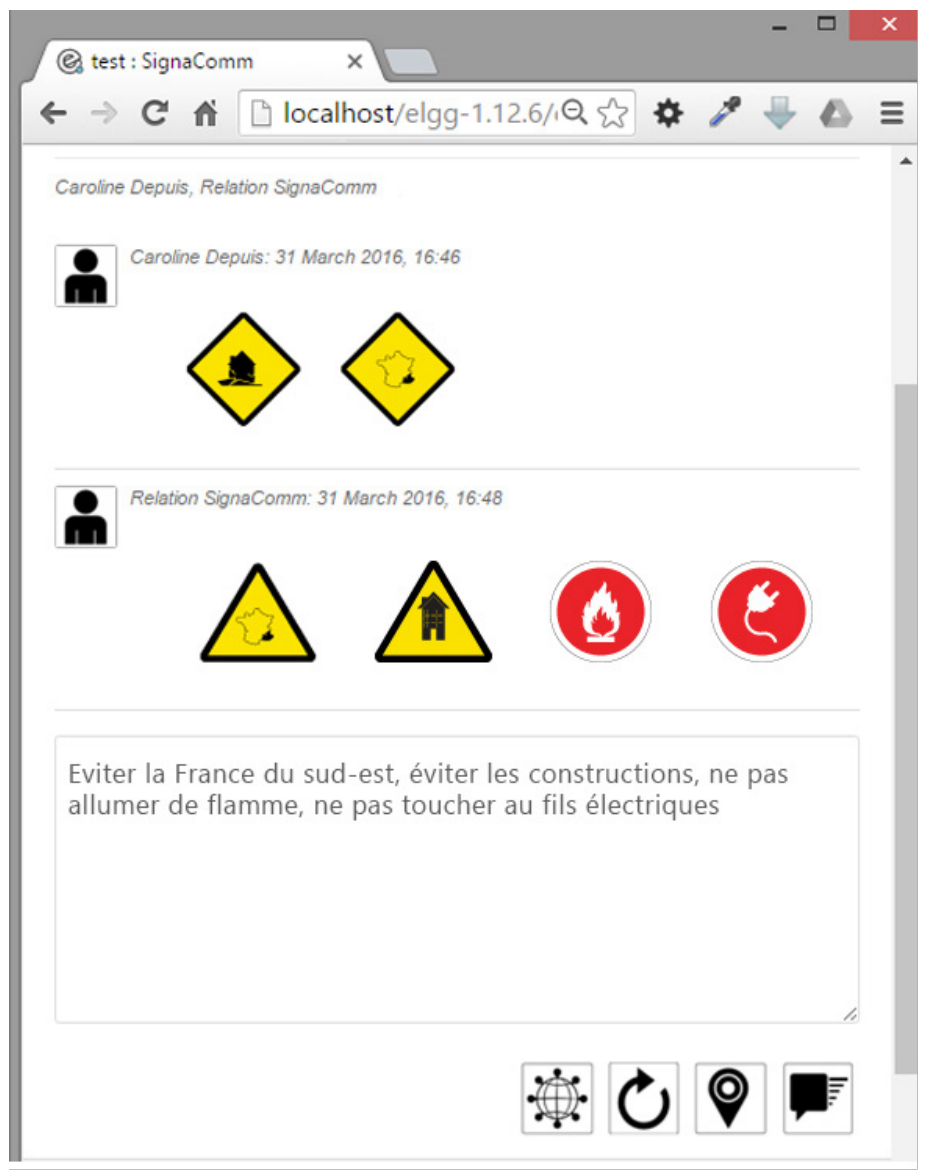

Figure 4 : exemple d'application de la page d'échange. Le sens de lecture des signagrammes est de haut en bas et de gauche à droit (Neurath, 1974)

En 2015, nous avons conçu et développé un premier logiciel pour traduire automatiquement un texte d'entrée en une séquence de signagrammes (Kordahi \& Baltz, 2015). Nous nous sommes appuyés sur la méthode de transfert sémantique de Emele et al. (2000) (figure 5). Ici, nous rappelons que le texte d'entrée provient d'un membre du SignaComm. Il est le texte source et est composé dans la langue préférée de l'utilisateur (Yang et al., 2010 ; Seme, 2001). Le résultat du 
texte d'entrée traduit en signagrammes est le « texte » cible. Les sorties $\mathrm{du}$ système de communication cible (la signalétique) proviennent du dictionnaire de signagrammes.

Le module de traduction du SignaComm exécute quatre tâches consécutives automatiquement qui sont aussi enregistrées dans sa base de données. La page HTML permet de recevoir le texte d'entrée du membre. Une requête transmet le texte d'entrée pour le faire traduire automatiquement en signagrammes vectoriels. Une nouvelle requête affiche le résultat de la traduction automatique dans la même page HTML. Et, une autre requête attend l'action du membre pour envoyer le message traduit au module de la messagerie, ou bien pour réinitialiser le processus de traduction automatique (figure 4).

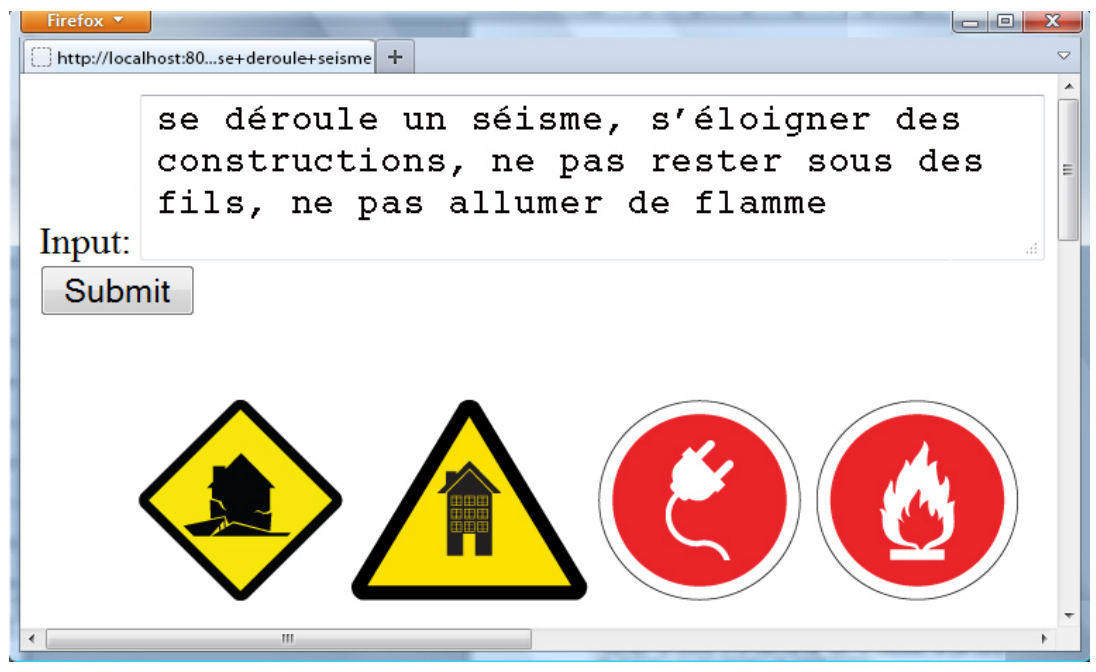

Figure 5 : exemple du résultat de la traduction automatique de syntagmes en signagrammes

La figure 4 montre un exemple d'application du SignaComm afin de traduire automatiquement une série de syntagmes et de les transmettre au membre correspondant. La figure 5 est prise de nos travaux (Kordahi \& Baltz, 2015, p. 24) pour représenter la traduction de consignes préventives (Prévention des risques majeurs). Le résultat de la figure 4 est comparable à celui de la figure 5 .

\subsection{Module de la messagerie instantanée}

Le module de la messagerie instantanée effectue deux tâches successives et automatiques qui sont enregistrées dans sa base de 
données. À travers le serveur, il reçoit du module de traduction les requêtes d'un membre sous forme de paquets, conformes à un protocole Internet commun, par exemple les paquets du Protocole de Transfert HyperTexte (HTTP) POST. Les paquets contiennent les informations de traduction (le message est traduit avant sa livraison) (Seme, 2001). La deuxième tâche sert à afficher l'historique des échanges entre les membres, dans la page HTML (figure 4).

\section{Discussion et conclusion}

Nous nous sommes efforcées de surmonter au moins deux contraintes. Premièrement, il n'est pas facile de représenter l'interface d'un RSN de communication à vocation internationale, touchant un public national et international. Nous avons donc proposé d'appliquer des règles pour tenter de résoudre cette difficulté, à savoir les principes de la conception universelle des interfaces numériques (Spiliotopoulos et al., 2012) et ceux de la création des signagrammes (Kordahi, 2013a).

Deuxièmement, lors d'une situation d'urgence, l'exactitude, la fiabilité et la rapidité de la transmission des consignes préventives sont nécessaires en vue d'aider les individus désavantagés. Nous avons donc proposé de composer le SignaComm de trois modules interreliés automatiquement (Parnas, 1972) et de les munir de bases données. Nous avons interrogé automatiquement les différents modules ainsi que leurs fichiers correspondants, et nous avons synchronisé l'échange d'informations entre eux. En testant techniquement le premier prototype du SignaComm, nous observons que le résultat de la traduction automatique est affiché correctement dans la page d'application et, ensuite, transmis en temps réel à son destinataire (figures 4 et 5).

Cependant, ce RSN présente une faiblesse. La compréhension de l'utilisation du SignaComm n'est peut-être pas immédiate dans tous les cas. Nous recommandons que le SignaComm devienne familier aux usagers, afin d'améliorer son utilisation durant le moment opportun. Une double approche serait intéressante à adopter. D'une part, une campagne marketing informative serait déployée en vue d'initier et de sensibiliser les usagers. Dans notre exemple d'application, les supports utilisés seraient ceux du Web, à savoir les sites gouvernementaux «Prévention des risques majeurs », microblogging de Twitter (Velve \& Zlateva, 2012). D’autre part, des prototypes seraient également rendus accessibles dans les diverses institutions spécialisées, publiques et 
d'enseignement (par exemple, UNESCO, la mairie, le lycée) afin d'encourager l'apprentissage spontané à travers l'expérimentation.

Enfin, dans un futur proche, nous tenterons d'améliorer ce premier modèle en suggérant deux directions de recherche. D'une part, nous proposerons de mener une étude qualitative et quantitative du SignaComm en le situant dans un contexte d'application simulé, qui est la signalisation participative de secousses sismiques. Ceci impliquerait qu'un public, situé dans des zones à risque, soit préalablement identifié dans la perspective de faire partie de cette étude, ou encore d'évoquer la possibilité de formes d'évaluations pair-à-pair par les participants. Il serait donc intéressant d'impliquer le Citoyen dans ces problématiques environnementales (Kaplan \& Marzloff, 2009).

D'autre part, nous souhaiterons examiner l'identité numérique des différents utilisateurs du SignaComm ainsi que les relations avec les catégories d'identité virtuelle (Turkle, 2011 ; Cardon, 2009 ; Kobsa et al., 2001). Cardon (2009) a proposé de " décomposer les différents traits identitaires que les plate-formes relationnelles demandent aux personnes d'enregistrer " (Cardon, 2009, p. 93) en différentes catégories d'identité visuelle. Ces catégories concernent les choix d'identification de l'internaute, comme le degré de partage d'informations personnelles, l'authenticité de l'identité de l'internaute, le type de contenus partagés. Nous prenons par exemple, le modèle de visibilité " clair-obscur » (Cardon, 2009). Dans cet environnement, les usagers rendent leur identité visible à leur réseau de proches (par exemple, les amis, les membres de famille, les collègues) afin d'être facilement accessibles. Il serait donc pertinent d'analyser ce contexte relationnel afin de tenter d'y situer le SignaComm.

\section{Remerciements}

Je remercie l'Université Paris 8 et le Laboratoire Paragraphe pour leur accueil chaleureux. Mes remerciements à Monsieur Claude Baltz, Professeur émérite en Sciences de l'Information et de la Communication à l'Université Paris 8 , ainsi qu'à Monsieur PierreMichel Riccio, Maître de recherche à l'École des Mines d'Alès. Je leur suis reconnaissante pour avoir examiné ce travail et de l'avoir enrichi de leurs observations. Merci également à Monsieur Houssam Naous, ingénieur en informatique et électronique, pour l'aide qu'il m'a apportée durant le développement de ce premier prototype. 


\section{Références}

Balagué, C., \& Fayon, D. (2012). Facebook, Twitter et les autres...: Intégrer les réseaux sociaux dans une stratégie d'entreprise. Pearson Education France.

Barnes, J. A. (1954). Class and Committees in a Norwegian Island Parish. New York: Plenum.

Barrington, L., Ricklin, N., \& Har-Noy, S. (2015). U.S. Patent No. 9,032,000. Washington, DC: U.S. Patent and Trademark Office.

Bell, P., Lai, C., Llewellyn, C., Birch, A., \& Sinclair, M. (2015). A System for Automatic Broadcast News Summarisation, Geolocation and Translation. In Sixteenth Annual Conference of the International Speech Communication Association.

Bertin, J. (1970). La graphique. Communications, 15(1), 169-185.

Ellison, N. B. (2007). Social Network Sites: Definition, History, and Scholarship. Journal of Computer Mediated Communication, 13(1), 210-230.

Cardon, D. (2009). Le design de la visibilité. Réseaux, (6), 93-137.

Desessard L., Massé M., Allain E., Vivien C., (2015), Code de la Route 2015, Paris : Dalloz-Sirey.

Di Gangi, P. M., \& Wasko, M. (2009). The Co-creation of Value: Exploring User Engagement in User-generated Content Websites. In Proceedings of JAIS Theory Development Workshop. Sprouts: Working Papers on Information Systems (Vol. 9, p. 50).

Di Mascio, T., Frigioni, D., \& Tarantino, L. (2010). VISTO: A new CBIR System for Vector Images. Information Systems, 35(7), 709-734.

Emele, M. C., Dorna, M., Lüdeling, A., Zinsmeister, H., \& Rohrer, C. (2000). Semanticbased Transfer. In Verbmobil: Foundations of Speech-to-Speech Translation (pp. 359-376). Springer Berlin Heidelberg.

Fernback, J. (1997). of Collective Principles. Virtual Culture: Identity and Communication in Cybersociety, 36.

« Fotolia », https://www.fotolia.com, dernière consultation le 22 novembre 2016.

Granovetter, M. (1983). The Strength of Weak Ties: A Network Theory Revisited. Sociological Theory, 1(1), 201-233.

Hartmann, F. (2008). Visualizing Social Facts: Otto Neurath's ISOTYPE Project. European Modernism and the Information Society: Informing the Present, Understanding the Past, 223-40.

Jain, R., Bose, J., \& Arif, T. (2013, December). Contextual Adaptive User Interface for Android Devices. In 2013 Annual IEEE India Conference (INDICON) (pp. 1-5). IEEE.

Kaplan, D., \& Marzloff, B. (2009). Pour une mobilité plus libre et plus durable (Vol. 1). FYP éditions.

Kordahi, M. (2016). L'ontologie sémantique: une application aux images vectorielles. Revue Management des Technologies Organisationnelles, (6), 92-107.

Kordahi, M., \& Baltz, C. (2015). La Traduction Automatique de Syntagmes en Signagrammes pour la prévention des risques. Revue Management des Technologies Organisationnelles, 5, 23-37.

Kordahi, M. (2013a). La signalétique comme nouveau système de communication artificiel. Canadian Journal of Information and Library Science, 37(4), 237-252.

Kordahi, M. (2013b). SignaNet: Premier dictionnaire électronique spécialisé de la signalétique. Pratiques et usages numériques H2PTM'2013, 385-387. 
Kordahi, M. (2012). La signalétique comme introduction à la communication internationale: perspectives d'automatisation (Doctoral dissertation, Paris 8).

Khanna, A. (2015). Facebook's Privacy Incident Response: A Study of Geolocation Sharing on Facebook Messenger. Harvard Dataverse, August.

Klinkenberg, J-M., (1996), Précis de sémiotique générale, De Boeck-Wesmael, Bruxelles.

Kobsa, A., Koenemann, J., \& Pohl, W. (2001). Personalised Hypermedia Presentation Techniques for Improving Online Customer Relationships. The Knowledge Engineering Review, 16(02), 111-155.

Lin, D., \& Ishida, T. (2014). User-centered Service Design for Multi-language Knowledge communication. In Serviceology for Services (pp. 309-317). Springer Japan.

Ma, C. M., Zhuang, Y., \& Fong, S. (2011, October). Information Sharing over Collaborative Social Networks Using xacml. In e-Business Engineering (ICEBE), 2011 IEEE 8th International Conference on (pp. 161-167). IEEE.

Mattelart, A. (2000). Vers une globalisation? Réseaux, 18(100), 81-105.

Neurath, M. (1974). Isotype. Instructional Science, 3(2), 127-150.

Parnas, D. L. (1972). On the Criteria to be Used in Decomposing Systems into Modules. Communications of the ACM, 15(12), 1053-1058.

"Prévention des risques majeurs ", http://www.gouvernement.fr/risques/seisme, dernière consultation le 22 novembre 2016.

Proulx, S. (2012). L'irruption des médias sociaux: enjeux éthiques et politiques. Médias sociaux: enjeux pour la communication, Québec, Presses de l'Université du Québec, 9-31.

Proulx, S., \& Latzko-Toth, G. (2000). La virtualité comme catégorie pour penser le social: l'usage de la notion de communauté virtuelle. Sociologie et sociétés, 32(2), 99-122.

Provitolo, D., Dubos-Paillard, E., \& Müller, J. P. (2009, January). Vers une ontologie des risques et des catastrophes: le modèle conceptuel. In Ontologie et dynamique des systèmes complexes, perspectives interdisciplinaires (pp. 1-16).

Rainie, H., \& Wellman, B. (2012). Networked: The New Social Operating System (p. 358). Cambridge, MA: Mit Press.

Rheingold, H., \& Lumbroso, L. (1995). Les communautés virtuelles. Addison-Wesley France.

Seme, Y. (2001). Method and System for Translating Instant Messages. U.S. Patent Application No. 10/035,085.

Spiliotopoulos, D., Tzoannos, E., Stavropoulou, P., Kouroupetroglou, G., \& Pino, A. (2012, July). Designing User Interfaces for Social Media Driven Digital Preservation and Information Retrieval. In International Conference on Computers for Handicapped Persons (pp. 581-584). Springer Berlin Heidelberg.

Takasaki, T., \& Mori, Y. (2007). Design and Development of a Pictogram Communication System for Children Around the World. In Intercultural Collaboration (pp. 193206). Springer Berlin Heidelberg.

Takasaki, T. (2006). PictNet: Semantic Infrastructure for Pictogram Communication. In The 3rd International WordNet Conference (GWC-06) (pp. 279-284).

Turkle, S. (2011). Life on the Screen. Simon and Schuster.

Vaillant, P. (1999). Sémiotique des langages d'icônes. 
Velve, D., \& Zlateva, P. (2012). Use of social media in natural disaster management. Intl. Proc. of Economic Development and Research, 39, 41-45.

Wandmacher, T., Antoine, J. Y., Poirier, F., \& Départe, J. P. (2008). Sibylle, an Assistive Communication System Adapting to the Context and its User. ACM Transactions on Accessible Computing (TACCESS), 1(1), 6.

Wellman, B. (2001, October). Little Boxes, Glocalization, and Networked Individualism. In Kyoto Workshop on Digital Cities (pp. 10-25). Springer Berlin Heidelberg.

Wellman, B., \& Gulia, M. (1999). Net Surfers Don't Ride Alone: Virtual Communities as Communities. Networks in the Global Village, 331-366.

Wong, Y., Grimm, S. M., Vera, N., Laverdet, M., Kwan, T. Y., Putnam, C. W., ... \& Little, C. (2012). U.S. Patent No. 8,271,260. Washington, DC: U.S. Patent and Trademark Office.

Yang, C. Y., \& Lin, H. Y. (2010, July). An Instant Messaging with Automatic Language Translation. In Ubi-media Computing (U-Media), $20103^{\text {rd }}$ IEEE International Conference on (pp. 312-316). IEEE. 